\title{
Relevance of the Surgical Care Improvement Project on glycemic control in patients undergoing cardiac surgery who receive continuous insulin infusions
}

\author{
Marie E. McDonnell, MD, ${ }^{\mathrm{a}}$ Sara M. Alexanian, MD, ${ }^{\mathrm{a}}$ Ana Junqueira, MD, ${ }^{\mathrm{a}}$ Howard Cabral, PhD, ${ }^{\mathrm{b}}$ and \\ Harold L. Lazar, MD ${ }^{\mathrm{c}}$
}

\begin{abstract}
Objective: The Surgical Care Improvement Project (SCIP) has benchmarked 6:00 AM blood glucose levels of less than $200 \mathrm{mg} / \mathrm{dL}$ on postoperative day (POD) 1 and 2 as quality measures of glycemic control in cardiac surgery. This study was undertaken to (1) determine the incidence of SCIP outliers in patients receiving a continuous insulin infusion (CII) targeted to maintain perioperative serum glucose levels less than $180 \mathrm{mg} / \mathrm{dL}$ after cardiac surgery, (2) identify the profile of patients who are SCIP outliers, (3) determine whether SCIP outliers have increased morbidity and mortality, and (4) identify more relevant benchmarks for glycemic control in patients having cardiac surgery.
\end{abstract}

\begin{abstract}
Methods: Between January 1, 2008, and April 30, 2011, a total of 832 patients underwent cardiac surgery and received CII to maintain serum blood glucose levels of less than $180 \mathrm{mg} / \mathrm{dL}$. Patients were divided into 2 groups: patients compliant with SCIP and SCIP outliers.
\end{abstract}

Results: The incidence of SCIP outliers was $6.6 \%$ (55/832). Patients more likely to be SCIP outliers had diabetes mellitus $(38,69 \%$ vs $250,32 \% ; P<.0001)$, a higher hemoglobin A1c $(8.74 \pm 2.25$ vs $7.59 \pm 2.90$; $P<.0009)$, and a higher body mass index $(31.1 \pm 6.5$ vs $29.2 \pm 5.7 ; P=.03)$. However, SCIP outliers had no increase in morbidity, mortality, or hospital length of stay.

Conclusions: Patients undergoing cardiac surgery may still be SCIP outliers despite CII targeted to maintain serum glucose levels below $180 \mathrm{mg} / \mathrm{dL}$; however, SCIP outliers had no increase in morbidity, mortality, or length of stay. (J Thorac Cardiovasc Surg 2013;145:590-7)

Perioperative glycemic control by maintaining serum glucose at less than $180 \mathrm{mg} / \mathrm{dL}$ in patients undergoing cardiac surgery has been found to reduce morbidity, mortality, and hospital length of stay, decrease the incidence of sternal wound infections, and enhance long-term survival and freedom from recurrent ischemic events. ${ }^{1-6}$ The Society of Thoracic Surgeons (STS) Practice Guidelines now recommend that all adults undergoing cardiac surgery, with and without diabetes mellitus, maintain serum glucose levels below 180 $\mathrm{mg} / \mathrm{dL}$ for the duration of their postoperative care. ${ }^{7}$

The Surgical Care Improvement Project (SCIP) is a national program undertaken to improve outcomes in surgery and, specifically, to reduce surgical site infections. ${ }^{8}$ One of the core SCIP quality measures involves glycemic control after cardiac surgery. SCIP has benchmarked that 6:00 AM

From the Section of Endocrinology, Diabetes, and Nutrition, ${ }^{\text {a }}$ Department of Biostatistics, ${ }^{b}$ and the Department of Cardiothoracic Surgery, ${ }^{\mathrm{c}}$ Boston Medical Center; and the Boston University School of Medicine, Boston, Mass.

Disclosures: Authors have nothing to disclose with regard to commercial support.

Read at the 92nd Annual Meeting of The American Association for Thoracic Surgery, San Francisco, California, April 28-May 2, 2012.

Received for publication March 29, 2012; revisions received Aug 16, 2012; accepted for publication Sept 13, 2012; available ahead of print Oct 11, 2012.

Address for reprints: Harold L. Lazar, MD, Department of Cardiothoracic Surgery, Boston Medical Center, 88 East Newton St, B402, Boston, MA 02118 (E-mail: harold.lazar@bmc.org).

$0022-5223 / \$ 36.00$

Copyright (c) 2013 by The American Association for Thoracic Surgery http://dx.doi.org/10.1016/j.jtcvs.2012.09.031 blood sugar levels on postoperative days (PODs) 1 and 2 be less than $200 \mathrm{mg} / \mathrm{dL}$ as the indicator of glycemic control after cardiac surgery. Compliance with these quality measures is publicly reported on the Centers for Medicare and Medicaid hospital website and is used to determine monetary reimbursement for participating hospitals. However, several studies have questioned the relevance of SCIP in directly leading to improved surgical outcomes. ${ }^{9-12}$ Furthermore, the relevance of a single SCIP glucose measurement in cardiac surgical patients already receiving a continuous insulin infusion (CII) targeted to achieve a serum glucose level of less than $180 \mathrm{mg} / \mathrm{dL}$ is unknown. This study was therefore undertaken to (1) determine the incidence of SCIP outliers in adults undergoing cardiac surgery and receiving a CII targeted to maintain perioperative serum glucose of less than $180 \mathrm{mg} / \mathrm{dL}$, (2) identify the profile of patients in this cohort who are SCIP outliers, (3) determine whether SCIP outliers already on a CII protocol have increased morbidity and mortality, and (4) identify more relevant benchmarks that can be used to determine glycemic control in patients undergoing cardiac surgery.

\section{METHODS}

This study was approved by the Boston University Medical Center Institutional Review Board (Protocol No. H-25760), and informed consent was obtained from each patient. 

Abbreviations and Acronyms
3BG = 3-day blood glucose
BMI = body mass index
$\mathrm{CABG}=$ coronary artery bypass surgery
$\mathrm{CII}=$ continuous insulin infusion
$\mathrm{HbA} 1 \mathrm{c}=$ hemoglobin A1c
ICU = intensive care unit
MHG = mean hospital glucose
POD = postoperative day
SCIP $=$ Surgical Care Improvement Project
STS $=$ The Society of Thoracic Surgeons

\section{Eligibility and Exclusion Criteria}

This was an observational, single-center study involving adult patients (both with and without diabetes) undergoing cardiac surgery from January 1, 2008, to August 30, 2011. Patients undergoing coronary artery bypass surgery (CABG), valve repair and replacement, and combinations of CABG plus valve surgery were included. Patients undergoing aneurysm repair with or without deep hypothermic circulatory arrest were excluded.

\section{Glycemic Control Protocols}

All patients received a CII (100 units of regular insulin in $100 \mathrm{~mL}$ of $0.9 \%$ normal saline) to maintain serum glucose between 120 and 180 $\mathrm{mg} / \mathrm{dL}$. Infusions were initiated on anesthetic induction and continued during the periods of cardiopulmonary bypass and cardioplegic arrest, after the discontinuation of cardiopulmonary bypass, and for at least 18 hours in the intensive care unit (ICU) as previously described. ${ }^{13}$ After the 18-hour ICU period, patients were transitioned off the CII using either short- or longacting insulin agents to maintain a fasting glucose level between 90 and $120 \mathrm{mg} / \mathrm{dL}$ and postsupper glucose level of less than $180 \mathrm{mg} / \mathrm{dL}$. Patients were transitioned off CII when they (1) maintained a stable intravenous insulin infusion rate for at least for 4 hours, (2) were extubated and off inotropic agents, and (3) were ready to begin oral, enteral, or parenteral nutrition. Serum glucose was measured every 30 minutes in the operating room and hourly in the ICU during CII. Blood glucose measurements were obtained using arterial catheters and finger sticks and were measured with the use of point-of-care glucose meters. The mean hospital glucose (MHG) measurement represents the average of all glucose values obtained during the hospital stay. Three-day blood glucose (3BG) values consisted of the average of all glucose values obtained from the end of anesthesia through the second POD (72 hours). SCIP glucose values represent the mean 6:00 AM glucose value for patients on POD 1 and POD 2.

\section{Operative Techniques}

Standard operative and anesthetic techniques were used in all patients. Myocardial protection was achieved with multidose infusions of antegrade and retrograde cold $\left(4^{\circ} \mathrm{C}\right)$ blood (hematocrit $\left.=20 \%\right)$ potassium $(28 \mathrm{mEq} /$ L) cardioplegia supplemented with mild systemic $\left(35^{\circ} \mathrm{C}\right)$ and topical (cold saline lavage at $4^{\circ} \mathrm{C}$ ) hypothermia. All vessels with at least a $50 \%$ stenosis were bypassed and each patient received at least 1 internal thoracic artery graft.

\section{Outcomes Measured}

In addition to parameters of glycemic control, major adverse events were also documented. These included 30-day mortality, the incidence of myocardial infarctions assessed by both enzyme and electrocardiographic changes as previously described, ${ }^{2}$ the incidence of cerebrovascular accidents, defined as a persistent neurologic deficit lasting 24 hours or more, the incidence of deep sternal wound infections, defined as any infection
TABLE 1. Patient profiles

\begin{tabular}{lccc}
\hline \multicolumn{1}{c}{ Variable } & $\begin{array}{c}\text { SCIP outlier } \\
(\mathbf{n}=\mathbf{5 5})\end{array}$ & $\begin{array}{c}\text { SCIP compliant } \\
(\mathbf{n}=\mathbf{7 7 7})\end{array}$ & $\boldsymbol{P}$ value \\
\hline Age (y) & $64 \pm 11$ & $66 \pm 12$ & .25 \\
Sex (M/F) & $39 / 16$ & $546 / 231$ & .78 \\
Hypertension (\%) & $48(87)$ & $662(85)$ & .17 \\
Diabetes (\%) & $38(69)$ & $250(32)$ & $<.0001$ \\
$\quad$ Insulin (\%) & $22(58)$ & $76(30)$ & $<.01$ \\
$\quad$ Oral (\%) & $16(42)$ & $133(53)$ & \\
$\quad$ Diet (\%) & $0(0)$ & $39(17$ & \\
Hyperlipidemia (\%) & $42(76)$ & $653(84)$ & .15 \\
Procedure & & & \\
$\quad$ CABG & $37(67)$ & $509(84)$ & .12 \\
CABG+ valve & $7(13)$ & $98(13)$ & \\
$\quad$ Valve & $11(20)$ & $170(22)$ & \\
Surgical class & & & \\
$\quad$ Elective & $22(40)$ & $373(48)$ & .24 \\
$\quad$ Urgent & $33(60)$ & $386(50)$ & \\
Emergency & $0(0)$ & $18(2)$ & \\
HbA1c & $8.74 \pm 2.25$ & $7.59 \pm 1.90$ & .0009 \\
Ejection fraction $(\%)$ & $49.0 \pm 14$ & $53 \pm 13$ & .10 \\
BMI (kg/m $\left.{ }^{2}\right)$ & $31.1 \pm 6.5$ & $29.2 \pm 5.7$ & .03 \\
\hline All
\end{tabular}

All values are mean \pm standard deviation. SCIP, Surgical Care Improvement Project; $M / F$, male/female; $C A B G$, coronary artery bypass graft; $H b A l c$, hemaglobin Alc; $B M I$, body mass index.

reaching or directly involving the sternum, and the incidence of atrial fibrillation that lasted longer than 15 minutes. All patients received postoperative beta-blockers and were continuously monitored on telemetry during their entire postoperative stay. In addition, secondary outcomes that were assessed included time on the ventilator (defined as time of admission to the ICU to the time of extubation), length of ICU stay, and length of hospital stay, all of which were standardized using fast-track protocols. Patients were also assessed for a clinical reason for SCIP "failure" by an endocrinologist intimately involved in glycemic control protocols.

\section{Statistical Analyses}

Data were summarized using the mean \pm standard deviation for continuous variables and the number and percentage for categorical variables using statistical analysis systems (SAS, version 9.0; SAS Institute, Inc, Cary, NC). SCIP compliance was determined prospectively by a hospital committee assigned to track 6:00 Am glucose on POD 1 and POD 2. All glucose levels were adjudicated prospectively in real time for accuracy and validated using retrospectively collected data. Comparison between SCIP compliant subjects and SCIP outliers was performed in bivariate analyses using 2-sample $t$ tests for continuous variables and $\chi^{2}$ or Fisher's exact tests for categorical data. Association of SCIP compliance was assessed with categorical surgical outcomes and continuous glucose outcomes (ie, 3BG) via odds ratios and $95 \%$ confident intervals using logistic regression analyses.

\section{RESULTS}

The results are summarized in Tables 1 to 3 and Figures 1 to 3 . The study population comprised 832 patients, 546 having isolated $\mathrm{CABG}, 105$ having $\mathrm{CABG}$ plus valve surgery, and 181 having isolated valve procedures. In this patient cohort, there were $55(6.6 \%)$ SCIP outliers (Table 1). Factors that did not predict SCIP failures included age, sex, ejection fraction, surgical class (elective, urgent, 
TABLE 2. Reasons for SCIP failure

\begin{tabular}{lc}
\hline \multicolumn{1}{c}{ Variable } & n (\%) \\
\hline Inadequate transition from CII to SQ insulin & $14(26.5)$ \\
Delay in initiating insulin infusion & $14(26.5)$ \\
No cause & $13(25)$ \\
CII protocol violation & $7(12)$ \\
Lapse in glucose monitoring & $4(8)$ \\
Insulin infusion stopped without transition to SQ insulin & $3(2)$ \\
\hline All values are mean \pm standard deviation. SCIP, Surgical Care Improvement Project; \\
$S Q$, subcutaneous; CII, continuous insulin infusion.
\end{tabular}

emergency), the presence of hypertension or hyperlipidemia, or the type of procedure performed. Three factors characterized patients who were SCIP outliers. They were more likely to have diabetes mellitus (38 [69\%] vs 250 [32\%]; $P<.0001)$ and be receiving insulin treatment (22 $[58 \%]$ vs $76[30 \%] ; P<.01)$. SCIP outliers showed higher preoperative hemoglobin A1c (HbA1c) levels indicative of poor glycemic control in the months preceding surgery $(8.74 \pm 2.25$ vs $7.59 \pm 1.90 ; P<.0009)$. In addition, they had significantly higher body mass indexes (BMIs; $31.1 \pm 6.5 \mathrm{~kg} / \mathrm{m}^{2}$ vs $29.2 \pm 5.7 \mathrm{~kg} / \mathrm{m}^{2} ; P=.03$ ). A logistic regression was used to determine the relationship between BMI and HbA1c. When applied to all subjects and controlling for serum creatinine level, BMI and HbA1c were both independently associated with being an SCIP outlier. However, when we isolated patients with previously diagnosed diabetes mellitus $(\mathrm{n}=288)$, while HbA1c remained significant, BMI was no longer a significant risk factor for failing the SCIP measure in this subgroup. Table 2 lists the reasons for SCIP failure. The most common causes were inadequate transition from CII to subcutaneous insulin $(14 ; 26.5 \%)$ and a delay in initiation of the insulin infusion $(14 ; 26.5 \%)$. In $13(25 \%)$ patients, no clear cause could be determined. Other factors included an infusion protocol violation $(7 ; 12 \%)$, a lapse in glucose monitoring $(4 ; 8 \%)$, and failure to transition to a subcutaneous insulin dose once the CII was terminated $(3 ; 2 \%)$.

Table 3 shows the outcomes of SCIP outliers. SCIP outliers had no significant difference in 30-day mortality, myocardial infarction, permanent strokes, deep sternal wound

TABLE 3. Postoperative outcomes

\begin{tabular}{lccr}
\hline \multicolumn{1}{c}{ Variable } & $\begin{array}{c}\text { SCIP outlier } \\
(\mathbf{n}=\mathbf{5 5})\end{array}$ & $\begin{array}{c}\text { SCIP compliant } \\
(\mathbf{n}=\mathbf{7 7 7})\end{array}$ & $\begin{array}{c}\boldsymbol{P} \\
\text { value }\end{array}$ \\
\hline Thirty-day mortality (\%) & $1(1.8)$ & $13(1.7)$ & .55 \\
Myocardial infarction (\%) & $1(1.8)$ & $11(1.4)$ & .52 \\
Permanent stroke (\%) & $1(1.8)$ & $7(0.9)$ & .39 \\
Deep sternal infection (\%) & $0(0)$ & $3(0.4)$ & 1.00 \\
Ventilatory support > 24 h (\%) & $5(9.0)$ & $52(6.7)$ & .38 \\
Multisystem failure (\%) & $1(1.8)$ & $7(0.9)$ & .43 \\
Atrial fibrillation (\%) & $9(16.3)$ & $235(30.3)$ & .05 \\
Hospital length of stay & $11.69 \pm 11.02$ & $9.75 \pm 7.83$ & .20 \\
\hline
\end{tabular}

All values are mean \pm standard deviation. SCIP, Surgical Care Improvement Project.
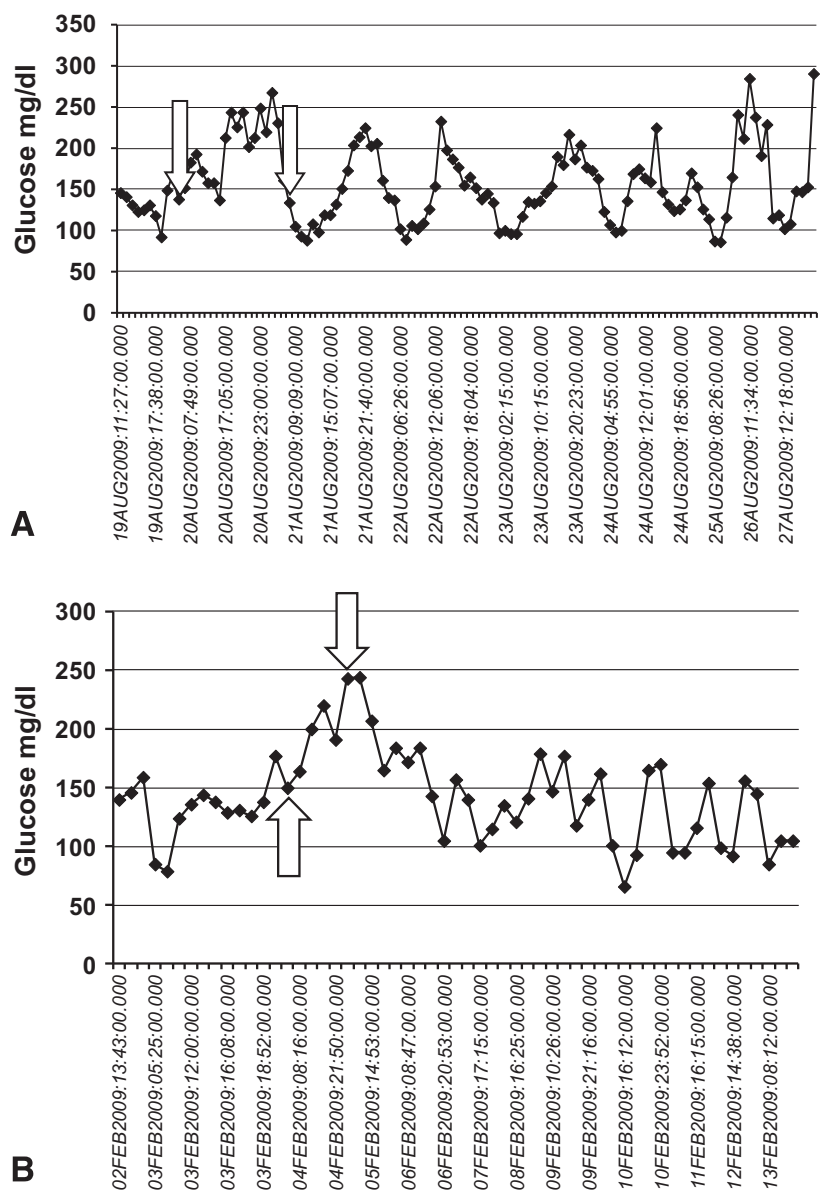

FIGURE 1. A, Surgical Care Improvement Project (SCIP) compliant. Hospital glucose values for an SCIP compliant patient. The arrows denote glucose values for 6:00 AM on the first and second postoperative days. Note that despite being SCIP compliant, a significant number of values were above The Society of Thoracic Surgeons and SCIP guidelines throughout the hospital stay. B, SCIP outlier. Hospital glucose values for a type 2 diabetic patient who was an SCIP outlier. The arrows denote glucose values for 6:00 AM on the first and second postoperative days. Note that despite being an SCIP outlier, the majority of glucose values were within The Society of Thoracic Surgeons and SCIP guidelines.

infections, ventilatory support for more than 24 hours, multisystem failure, or hospital length of stay. The incidence of atrial fibrillation appeared to be less in patients who were SCIP outliers $(9,16.3 \%$, vs $235,30.3 \% ; P=.05)$, even after controlling for $\mathrm{HbA} 1 \mathrm{c}$ and serum creatinine.

Figure 1, $A$ and $B$, shows the limitations of SCIP in determining glycemic control. Figure $1, A$, shows the serum glucose values in a patient who is SCIP compliant on PODs 1 and 2. However, as shown in the figure, a significant percentage of these values exceeded both STS and SCIP guidelines throughout the postoperative period. Figure 1, $B$, shows glucose values for a patient with type 2 diabetes not compliant with SCIP on POD 2. Nevertheless, this patient maintained both the STS and SCIP guidelines for the 


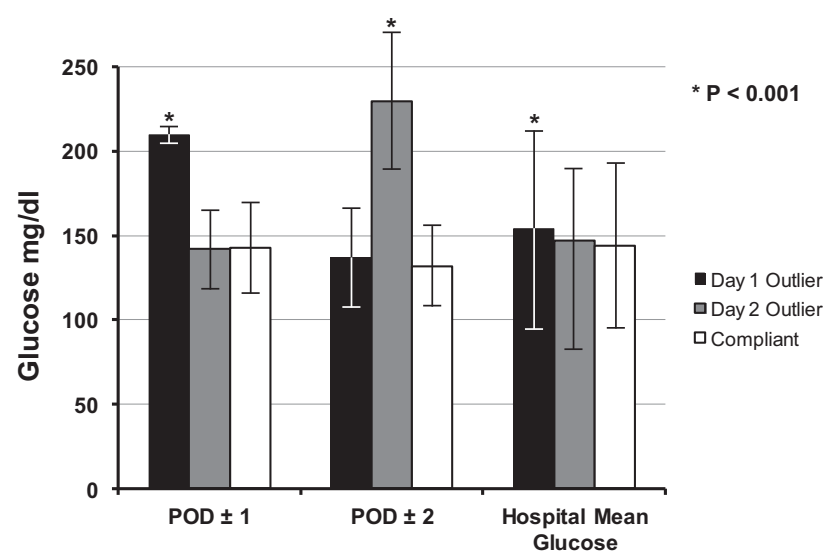

FIGURE 2. Surgical Care Improvement Project versus hospital mean glucose. Day 1 outliers were compliant on day 2. Day 2 outliers were compliant on day 1. All groups have hospital mean glucose values that are within The Society of Thoracic Surgeons and Surgical Care Improvement Project guidelines. $P O D$, Postoperative day.

remainder of the hospital stay. The limitations of SCIP as a measure of glycemic control are further highlighted in Figure 2. Glucose levels at 6:00 AM in SCIP outliers on POD 1 decreased to compliant levels on POD 2 $(210 \pm 5.6 \mathrm{mg} / \mathrm{dL}$ vs $137 \pm 28 \mathrm{mg} / \mathrm{dL} ; P<.001)$. Patients who were SCIP outliers on day 2 had compliant glucose levels on day $1(230 \pm 39 \mathrm{mg} / \mathrm{dL}$ vs $142 \pm 23 \mathrm{mg} / \mathrm{dL}$; $P<.001)$. MHG showed that although SCIP outliers on day 1 had significantly higher glucose levels than SCIP outliers from day 2 and SCIP compliant patients, MHG was consistent with STS and SCIP guidelines $(154 \pm 61$ $\mathrm{mg} / \mathrm{dL}$ SCIP outliers day $1 ; P<.001 ;$ vs $147 \pm 56 \mathrm{mg} / \mathrm{dL}$ SCIP outliers day 2 , and $144 \pm 48 \mathrm{mg} / \mathrm{dL}$ SCIP compliant). When early postoperative glycemic control using the $3 \mathrm{BG}$ index was assessed (Figure 3), SCIP outliers were also consistent with STS and SCIP guidelines, although patients noncompliant with SCIP continued to show higher glucose values $(162 \pm 19 \mathrm{mg} / \mathrm{dL}$ vs $141 \pm 6 \mathrm{mg} / \mathrm{dL} ; P<.001)$.

Overall, 430 or approximately half of the entire cohort had at least 1 glucose value less than $70 \mathrm{mg} / \mathrm{dL}$ during the course of their entire hospital stay. There was no difference in the incidence of hypoglycemia between SCIP outlier and SCIP compliant patients. Among the patients who did experience hypoglycemia, this represented only $1.2 \%$ of all their hospital glucose values. There was no associated morbidity in any of the patients in whom hypoglycemia developed.

\section{DISCUSSION}

Our study has shown that in patients undergoing cardiac surgery and receiving CII to maintain a serum glucose level of less than $180 \mathrm{mg} / \mathrm{dL}$, a certain percentage of patients will be SCIP outliers. Outliers are more likely to have diabetes

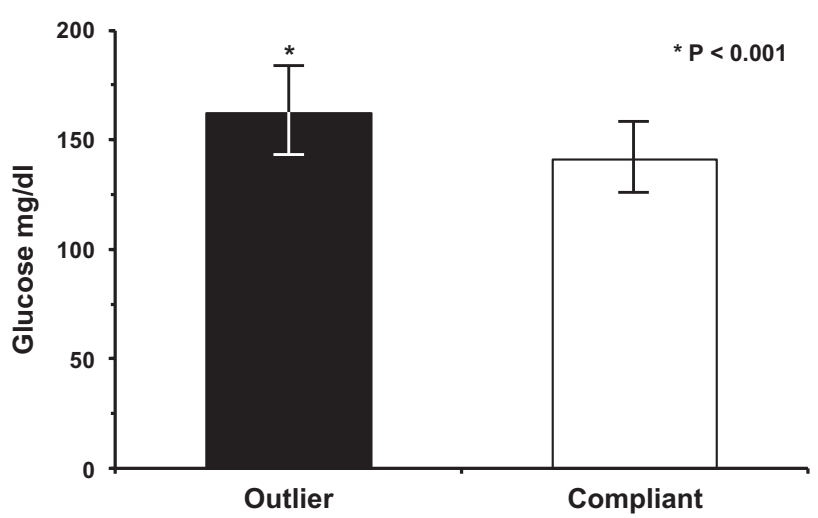

FIGURE 3. Three-day blood glucose. Surgical Care Improvement Project (SCIP) outliers have higher 3-day blood glucose values than SCIP compliant patients but are well within The Society of Thoracic Surgeons and SCIP guidelines.

necessitating insulin, have higher HbAlc levels, and increased BMI. Despite being SCIP outliers, these patients had no increase in postoperative morbidity, mortality, or length of hospital stay. Similar results were found by Murphy and coworkers, ${ }^{9}$ who studied the incidence of SCIP compliance in 108 patients undergoing cardiac surgical procedures. Although each patient received CII designed to maintain serum glucose levels between 100 and 140 $\mathrm{mg} / \mathrm{dL}, 10(9 \%)$ patients were found to be SCIP outliers. Glycemic control was assessed by the average blood glucose in the initial 48 hours postoperatively, the duration of hyperglycemia (percentage of time with blood glucose $>200$ $\mathrm{mg} / \mathrm{dL}$ ), and the intensity of the implementation of the insulin infusion (defined as the number of blood glucose checks per hour on the insulin infusion). The average mean blood glucose between the SCIP compliant patients and outliers was $148 \pm 2$ versus $203 \pm 10 \mathrm{mg} / \mathrm{dL}(P<.0001)$. However, the postoperative percentage of time that was spent with a blood glucose level of more than $200 \mathrm{mg} / \mathrm{dL}$ was higher in SCIP compliant patients $(14 \% \pm 0.2 \%$ vs $0.48 \% \pm 4 \%$; $P<.0001)$. The number of glucose checks per hour tended to be higher in the noncompliant group, indicating that SCIP noncompliance was not due to less intense implementation of the insulin infusion protocol. Murphy and coworkers ${ }^{9}$ concluded that even the use of CII targeted to a blood glucose of less than $140 \mathrm{mg} / \mathrm{dL}$ did not guarantee compliance with SCIP even though more frequent adjustments were made in the SCIP outlier group. Stulberg and coworkers ${ }^{10}$ sought to examine the relationship between SCIP adherence and postoperative infection rates in a retrospective study using the Premier Prospective database. This involved 398 hospitals and 405,720 patients. The incidence of surgical infections was $1.1 \%$ in SCIP compliant patients versus $1.5 \%$ in noncompliant patients. The authors were not able to demonstrate a relationship between SCIP adherence and a decreased incidence of infections. This is in agreement 
with other studies that have failed to validate the effectiveness of SCIP in reducing infection rates. ${ }^{11,12}$

It is not surprising that the 2 isolated SCIP glucose measurements do not predict postoperative outcomes. Studies have shown that metrics that incorporate glucose values over longer time periods are prognostically superior to data from just the first 24 or 48 hours of a patient's hospitalization. Furnary, $\mathrm{Wu}$, and Bookin ${ }^{14}$ assessed the efficacy of glycemic control using 3BG in 1980 patients undergoing cardiac surgery using CII. This was calculated from an average of all glucose values on the day of surgery and the first and second PODs. An increase in 3BG was an independent predictor of postoperative mortality $(P<.001)$. Changes in 3BG were also predictive of deep sternal wound infections, longer hospital lengths of stay, the need for blood transfusions, new-onset atrial fibrillation, and low cardiac output syndrome. Kosiborod and coworkers ${ }^{15}$ also sought to determine which metrics of glycemic control would best predict hospital mortality in patients admitted with an acute myocardial infarction. In this study, 3 parameters of glycemic control were compared with a single admission glucose value in the ability to predict hospital mortality. MHG was the average of each patient's glucose value during the hospitalization. A time-averaged glucose value was the area under the curve of all glucose values during a specified time period divided by the length of the time period. The hyperglycemic index was the area under the curve of hyperglycemic glucose values over a particular time period. ${ }^{16}$ Each of these methods was evaluated over the first 24 hours, 48 hours, and for the entire hospitalization, and they were better predictors of hospital mortality than a single admission glucose level. MHG over the entire hospital stay was found to be the easiest and most practical parameter to assess glycemic control and its relationship to hospital survival. The authors concluded that metrics that incorporated glucose values over longer time periods were prognostically superior to a single glucose value or data obtained from just the first 24 or 48 hours of a patient's hospitalization after an acute myocardial infarction.

Achieving glycemic control in the perioperative period requires a multidisciplinary approach that includes representatives from nursing, anesthesiology, pharmacy, surgery, and endocrinology. At our own institution, we formed a Perioperative Glycemic Control Committee to help meet SCIP guidelines. A representative from this committee receives SCIP outcome data on a monthly basis and reviews each case that does not meet the glycemic target. Committee members then provide direct feedback to the individual disciplines to make necessary adjustments or identify areas of deficiencies.

Our study is limited in that it was retrospective and we were not able to assess in real time the causes for SCIP failure. Although we did review in detail the hospital records for all patients with SCIP failure, we may not have been able to account for any undocumented changes in clinical conditions that may have also been responsible for SCIP outliers.

Our own data corroborate the findings of these studies that have shown that isolated serum blood glucose values do not accurately predict patient outcomes and do not adequately represent glycemic control during the hospital stay. Therefore, failure to meet SCIP glucose guidelines should not imply substandard care for these patients having cardiac operations. Adherence to SCIP glycemic values is only 1 aspect of effective patient care. Morbidity and mortality in patients undergoing cardiac surgery are likely to be influenced by other factors independent of glycemic control. Hence, SCIP benchmarks for glycemic control should not be the only metric for reporting operative outcomes and determination of hospital monetary reimbursements.

\section{References}

1. Furnary AP, Gao G, Grunkemeier GL, Wu Yx, Kerr KS, Bookin SO, et al. Continuous insulin infusion reduces mortality in patients with diabetes undergoing coronary artery bypass grafting. J Thorac Cardiovasc Surg. 2003;125:1007-21.

2. Lazar HL, Chipkin SR, Fitzgerald CA, Bao Y, Cabral H, Apstein CS. Tight glycemic control in diabetic coronary artery bypass graft patients improves perioperative outcomes and decreases recurrent ischemic events. Circulation. 2004; 109:1497-502.

3. Zerr KJ, Furnary AP, Grunkemeier GL, Bookin S, Kanhere V, Starr A. Glucose control lowers the risk of wound infection in diabetics after open heart operations. Ann Thorac Surg. 1997;63:356-61.

4. van den Berghe G, Wouters P, Weekers F, Verwaest C, Bruyninckx F, Schetz M, et al. Intensive insulin therapy in critically ill patients. $N$ Engl J Med. 2001;345: 1359-67.

5. D'Alessandro C, Leprince P, Golmard JL, Ouattara A, Aubert S, Pavie A, et al. Strict glycemic control reduces EuroSCORE expected mortality in diabetic patients undergoing myocardial revascularization. J Thorac Cardiovasc Surg. 2007; 134:29-37.

6. Hruska LA, Smith JM, Hendy MP, Fitz UL, McAdams S. Continuous insulin infusion reduces infectious complications in diabetics following coronary surgery. J Card Surg. 2005;20:403-7.

7. Lazar HL, McDonnell MM, Chipkin SR, Furnary AP, Engelman RM, Sadhu AR, et al. The Society of Thoracic Surgeons Practice Guidelines Series: Blood glucose management during adult cardiac surgery. Ann Thorac Surg. 2009;87:663-9.

8. Centers for Medicare and Medicaid Services, Joint Commission. http://www. jointcommission.org/performancemeasurement/current + nhgm + manual.htn.

9. Murphy MA, Whitman I, Campfield A, Moxey E, Haddad M, Whitman G. Intense implementation of a strict insulin infusion protocol does not guarantee postoperative glycemic control. J Am Coll Surg. 2010;211:465-9.

10. Stulberg JJ, Delaney CP, Neuhauser DV, Aron DC, Fu P, Koroukian SM. Adherence to surgical care improvement project measures and the association with postoperative infections. JAMA. 2010;303:2479-85.

11. Hawn MT, Itani KM, Gray SH, Vick CC, Henderson W, Houston TK. Association of timely administration of prophylactic antibiotics for major surgical procedures and surgical site infection. J Am Coll Surg. 2008;206:814-9.

12. Nguyen N, Yegiyants S, Kaloostian C, Abbas MA, Difronzo LA. The Surgical Care Improvement Project (SCIP) initiative to reduce infection in elective colorectal surgery: which performance measures affect outcome? Am Surg. 2008;74: 1012-6.

13. Lazar HL, McDonnell MM, Chipkin S, Fitzgerald C, Bliss C, Cabral H. Effects of aggressive versus moderate glycemic control on clinical outcomes in diabetic coronary artery bypass graft patients. Ann Surg. 2011;254:458-64.

14. Furnary AP, Wu Y, Bookin S. Effect of hyperglycemia and continuous intravenous insulin infusions on outcomes of cardiac surgical procedures: The Portland Diabetic Project. Endo Prac. 2004;10(Supp 2):21-33.

15. Kosiborod M, Inzucchi SE, Krumholz HM, Xiqo L, Jones PG, Fiske S, et al. Glucometrics in patients hospitalized with acute myocardial infarction: defining the optimal outcomes-based measure of risk. Circulation. 2008;117:1018-27.

16. Vogelzang M, Van der Horst IC, Nigesten MW. Hyperglycemic index as a tool to assess glucose control: a retrospective study. Crit Care. 2004;8:12122-7. 


\section{Discussion}

Dr Anthony P. Furnary (Portland, Ore). Dr McDonnell, thank you for a beautiful and very concise presentation. We can all be frustrated by this SCIP measure. I think all in this room can agree that the SCIP benchmarks that have been put forth are really scientifically unfounded, and, as you have said, inaccurate. To me, they make little sense. As you have shown, being an outlier at 6:00 AM on the first or second day really has nothing at all to do with the glycemic state of the patient in the first 3 days.

Just as the SCIP glycemic benchmarks have nothing to do with glycemic state, I think it is important to note that tight glycemic control has nothing to do with cardiac surgery outcomes in patients who do not have diabetes. This is a really important point. I want to repeat it. There has not been a single study showing that tight glycemic control alters cardiac surgery outcomes in patients who do not have diabetes. The only outcomes benefits that have ever been shown are in patients who have diabetes or in surgery patients who are receiving total parenteral nutrition for more than 5 days.

A lot of authors have missed that important caveat. It is important because it comes as no surprise, then, that any study that combines both patients with diabetes and those without diabetes in its study population and looks at glycemia-related outcome end points will find that those glycemia-related outcomes are preordained to be what everybody now calls "noninferior." Thus it does not matter whether you look at target blood glucose level, moderate versus tight glucose control, or a glycemic SCIP measure or you look at actual achieved 3BG; you will always end up with a noninferior outcome.

Furthermore, in patients with diabetes, the achieved blood glucose levels are always either at the upper end of the target blood glucose range or, more frequently above that target range. If you wanted to get $100 \%$ compliance within a target, say you wanted to be $100 \%$ less than $180 \mathrm{mg} / \mathrm{dL}$, then you would have to target $150 \mathrm{mg} / \mathrm{dL}$ to get everybody there, to less than $180 \mathrm{mg} / \mathrm{dL}$. I have 3 questions.

First, did you get a chance to analyze the outcomes of just your diabetes subset of patients with regard to $3 \mathrm{BG}$ and SCIP compliance?

Dr McDonnell. Thank you very much, Dr Furnary, for that excellent summary. I agree with the majority of your points. You are absolutely right that the majority of the available prospective data in group of patients who are newly revascularized supports the concept that glycemic control reduces the excess risk seen in patients with diabetes having CABG surgery. Not all data are consistent with this benefit being unique to patients with diabetes, however, and subgroup analyses of larger studies suggest CABG patients without diabetes may benefit substantially from postoperative intensive insulin therapy.

I did look at the diabetes CABG subset. This represented $28 \%$ of the entire cohort, so it was a small subset. Just of note, $75 \%$ of the operations in this data set were CABG and $37 \%$ of the total group had a prior diagnosis of diabetes.

We found that $3 \mathrm{BG}$ was almost the same between the patients with diabetes and those without diabetes, $148 \mathrm{mg} / \mathrm{dL}$ versus 135 $\mathrm{mg} / \mathrm{dL}$, respectively, reflecting that all patients receive the same glycemic care perioperatively. We did not see a difference in outcomes between these groups, presumably for 2 reasons: (1) the glucose difference was minimal; (2) the event rate was low, as would be expected in a group of this size in many centers. Our previous speaker noted that the mortality reduction seen over the past 10 or 15 years in cardiac surgery is profound depending on the data set you look at, and it is now difficult to show a difference in outcomes owing to any single intervention, certainly in mortality.

Dr Furnary. As you know, we have developed the 3BG because it covers every component of glycemia-related risk throughout the postoperative period. SCIP decided not to use 3BG or not to use any composite outcome for several reasons: (1) a composite outcome is difficult to collect; (2) it is complex to collect; and (3) it is costly to collect. What would your recommendation be to SCIP? Furthermore, what is your recommendation for the STS in regard to glycemic metrics for patients having cardiac surgery? It is my opinion that the STS really ought to be in charge of this and define a glycemia-related metric for all patients requiring cardiac surgery-and use one that makes scientific sense. What should we collect in the STS database and what should we tell SCIP that they ought to collect for some sensible scientifically related composite glucose measurement?

Dr McDonnell. Thank you for your question. First of all, I am pleased to be part of this conversation, because I think that it is not an easy answer and we need to discuss it across all involved disciplines. I would be happy, as would many of my colleagues, with a measurement that involved more than 1 or 2 measurements to arrive at a true mean and specifically avoid isolated measurements, which are not valid as tools to assess overall glycemic control. As an endocrinologist, I find it concerning that we are using isolated measurements to judge the glycemic care of our patients having cardiothoracic surgery. SCIP could potentially calculate 3BG on a subset of patients randomly selected and report that in the form of an audit, or we could collect 4 to 8 measurements over the course of 2 to 3 days. The SCIP monitors may have difficulty with these more complex measures inasmuch as it would increase time required to collect data, and that would come at a cost. However, the conversation needs to continue, and, I agree, STS needs to be in the forefront.

Dr Furnary. Last question: We know that $75 \%$ of the SCIP "violations" (I call them violations) were caused by a delayed start, poor transition from intravenous to subcutaneous, or some other protocol violation, and the remaining ones were caused just because they were on the protocol and were in that group of patients who end up higher than the protocol target. We also know that the actual achieved glucose is often higher than the target. Shouldn't we therefore just all lower our upper target range and to say 150 $\mathrm{mg} / \mathrm{dL}$ and keep all our patients on insulin drip protocols for 3 days instead of just 18 hours? That way, all of the glycemia-related risks are fully covered. This is what we have done in Portland, and we end up having $100 \%$ SCIP compliance on this glycemic measure, so it is not so frustrating anymore. We just forget about the SCIP thing, because it always works.

Dr McDonnell. I will be frank that it took us time, but we have found that many of the elements related to SCIP failure that I noted are remediable. We have remediated many of them in the past 1 to 2 years so that we are at $100 \%$ compliance for most months over the past year. We have been over $95 \%$ for 2 years. The goal can be reached in most patients inasmuch as insulin is certainly effective. However, reaching the SCIP goal, as we have shown, does not have significant meaning for the patients. Of importance here is 
that SCIP compliance should reflect overall glycemic control and not merely an attempt to arrive temporarily at the 6:00 AM target of less than $200 \mathrm{mg} / \mathrm{dL}$, after which control may be abandoned.

What I would also say is that intravenous insulin therapy that is prolonged after cardiothoracic surgery with subcutaneous insulin to cover meals is very elegant and is a beautiful therapy. However, I just do not think it is available to every institution. For many reasons, we decided to master the subcutaneous transition by day 2 , and that involved a team of providers, including me and another attending, with fellows, pharmacists sometimes, and also midlevel providers, who can be specifically trained to manage intensive subcutaneous insulin in patients having cardiothoracic surgery. I would say either way it is accomplishable, and whether you are doing a prolonged insulin infusion on the step-down unit or early transition to scheduled subcutaneous insulin, an enormous amount of commitment to both the nurses and to patients is required, and you know that better than anyone else.

Dr Lars G. Svensson (Cleveland, Ohio). Dr McDonnell, congratulations on a very important and timely study. Unfortunately, this is something that we are going to have to deal with increasingly, as you, I am sure, are all too aware. At the moment, there are some 70 patient and outpatient measures that are imposed on institutions, and at the last count I saw, they are going up to about 120. Increasingly, we are going to be playing defense against all these various quality measures, and it certainly is a challenge for those that have to deal with responding to this and observing the quality of our departments.

As you know, the bump-up in sugar after surgery is a normal response to trauma. We all learned that in physiology, and our trauma surgery colleagues have had to deal with the same issue. Unfortunately, getting the SCIP measures changed has been very difficult. As you know, the time interval, which is now measured, will be changing. Fortunately, for those of you who had to deal with the issue of cold patients in the ICU, we managed to convince various people that the SCIP measure for that was completely wrong, that we would be doing patients harm if we rewarm them after surgery to the point that they are normothermic with the pump. So they did respond to that.

In response to the issue of blood sugars, we went through various changes to try to deal with this issue. We noticed that blood sugar levels rose in patients who received vancomycin with a glucose carrier; patients who were sicker, who usually went up to the ICU late in the day or were on epinephrine and also had an increase in their blood sugar levels. So we recommended various things, and our nurses were very helpful. For example, a 10 o'clock glucose value that rises above $160 \mathrm{mg} / \mathrm{dL}$ we recheck at 3 o'clock to try to meet this very measure. The danger in this measure aggressively is particularly that the patients could become hypoglycemic.

I have a couple of questions. The categories of complications that you looked at were fairly broad. The ones as a surgeon I would worry about as potentially being a factor with high sugars and diabetes is in the patients with bilateral internal thoracic arteries and the risk of wound infection. We know they have a higher risk. Do you have the numbers to look at that subcategory of patients? Your incidence of multiple organ failure was remarkably good and low, but that may be another place where high sugars may play a role as also with lactic acidosis. Did you look at these subgroups?
My final question concerns talk about a National Institutes of Health study within the cardiothoracic cooperative network looking at this very issue. Is this something that you think should be taken further?

Dr McDonnell. Thank you very much. I echo your sentiment and your comments about the strategies that you have used to improve or to reach the SCIP measure and your concern for the potential to go overboard with targeted insulin therapy to attain a glucose goal, placing patients at risk.

To answer your first question, we do not have the graft type in this data set, specifically the bilateral internal thoracic arteries, but we have discussed this as a variable that may be significant.

Your second question concerned the fact that SCIP is linked to monetary reimbursement and to judging providers and their care. I think it should go to wherever committee or organization it could go to in order to make rationale change. Larger multi-institutional studies are costly, but if they can be used to assess the relevance of several SCIP measures, it may be of value. In my experience and opinion, once a program has made the effort to control glucose and if this can be proven or established by the institution, it is frustrating to have to address the random miss, the random SCIP outlier who is not at increased risk of poor outcomes. Unfortunately, at our institution we continue to have to respond to SCIP reviewers by spending up to an hour per case trying to determine and document why a patient had a glucose elevation for a few hours of their entire stay, which likely has no relevance to their care. Additionally, we are asked how to prevent this from happening, which, as we have shown, is impossible to say for all patients inasmuch as in at least $25 \%$ of cases there is no identifiable reason for the hyperglycemic event. This is consistent with the idea that transient physiologic hyperglycemia can be unpredictable after cardiothoracic surgery surgery, which, despite having little to no clinical consequence, currently has significant administrative consequence.

Dr Thomas M. Beaver (Gainesville, Fla). I would caution against totally throwing SCIP out. I believe there is a "culture effect" in trying to make things better. I would also remind the audience that glucose is just one of the many SCIP measures, including getting the antibiotics in on time during the time out with the anesthesiologist; stopping the antibiotics; appropriate choice of antibiotics; and warming patients. You can also look at the SCIP overall composite score. What we found at our own institution is that, as we have improved our SCIP composite score, our rate of global class I surgical infections have decreased by $50 \%$ across the board.

Have you had a chance to look at your SCIP composite score in this group of patients to determine whether that may have had any effect?

Dr McDonnell. Thank you for your comment. I think that is such an important way to end this session. SCIP is important in the way it improved glycemic control and other evidence-based practice in many institutions. Institutions should be held accountable for their practice of evidence-based care, and this is unquestionable for the sustained achievement of optimal patient outcomes. The priority with SCIP continues to be in line with clinicians, which is to optimize clinical outcomes. What we and others have tried to highlight, however, is that the parameters selected have to be simple to measure as well as evidence-based and at minimum reflective of the actual practice. Otherwise, the SCIP culture becomes a negative force as an unintended consequence. 
Regarding the composite measure, it is possible that Dr Lazar knows the answer to this. I do not know whether the composite score for cardiothoracic surgery has been linked to outcomes in our institution. I do know that we have extremely low infection rates, as many hospitals do now, after the institution of many
SCIP measures, not just glucose control. But I do not know whether Dr Lazar wants to comment on that.

Dr Lazar. In brief, the answer is no, we have never ever been able to correlate any of the SCIP measures with infection in our institution. 